\section{A difference detection system for high precision measurements of ultrafast transmission changes}

\author{
H J Polland and W Zinth \\ Physik Department der Technischen Universität München, \\ München, Germany
}

Received 7 November 1984, in final form 10 December 1984

\begin{abstract}
Ultrafast transmission changes can be recorded with high precision by means of a difference detection system. Using single pulses of low energy $(0.1 \mathrm{~nJ})$ and low repetition rate, variations of the transmitted energy induced by an excitation pulse are measured with an accuracy of $10^{-4}$.
\end{abstract}

\section{Introduction}

In recent years numerous ultrafast processes in physics, chemistry, and biology have been studied with the help of picosecond and femtosecond optical pulses. Frequently, excite and probe experiments were applied. One ultrashort light pulse is split into two parts. A strong component excites the sample, whereas the weaker component, delayed in time by an optical delay line, probes the sample at the delay time $t_{\mathrm{D}}$. Measuring the transmission of the sample as a function of delay time one obtains information on the dynamical processes in the sample. In many cases the time dependent transmission changes are small, e.g. when the sample is photochemically unstable, allowing only slight excitation. As a consequence the transmission changes of the sample must be determined with high precision.

In general, the incoming light energy and the energy of the transmitted pulse are recorded with the help of two photodetectors and $A / D$ converters with high linearity. It is evident that the precision of the measurement depends on the linearity and the dynamic range of the detectors and $A / D$ converter system. Using this measuring system in conjunction with picosecond pulses from a Nd-glass laser system, one normally obtains a precision of $0.5 \%-1 \%$. In this paper we present a difference detection system which allows the detection and measurement of much smaller changes of transmission. The sensitivity is improved by a factor of 50 when replacing the conventional photodetectors by a suitably designed difference detector system. Transmission changes as small as $10^{-4}$ can be measured with single picosecond light pulses from a lowrepetition rate laser system.

\section{The optical set-up}

The schematic of the optical set-up used to measure weak transmission changes is shown in figure 1. A single pulse from the picosecond laser system is divided into a weak probing pulse, no 1 , and a strong exciting pulse, no 2 . The probing pulse is delayed in an optical delay line and split into two parts by the beam splitter $\mathbf{B S}^{\prime}$. The two probing beams travel through the sample and are imaged onto two photodiodes of the difference detector. The detector records the difference between the light energy falling onto the two photodiodes, i.e. in the present arrangement it measures the difference in transmitted energies

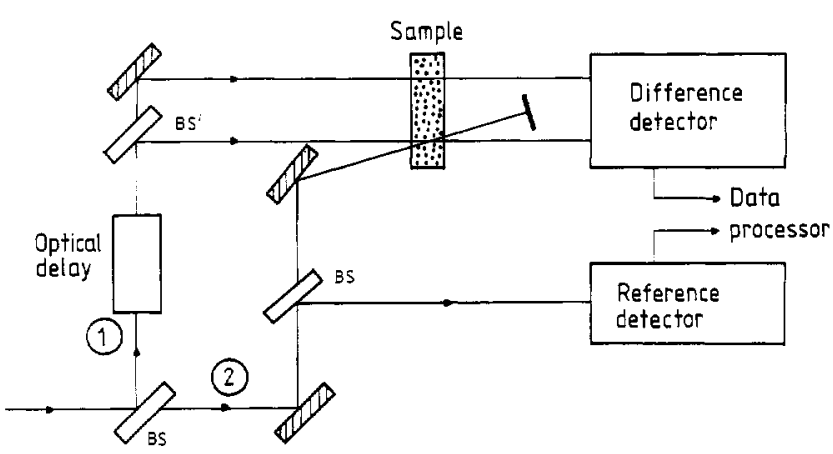

Figure 1. Optical set-up for the measurement of weak time-dependent transmission changes using the difference detection system with the optical (prism) delay allowing delays in the picosecond time domain and with the beam splitters BS and BS'.

of the two probing beams. Since the exciting pulse (no 2 in figure 1) passes the sample only in the region of one probing beam, the difference detector will measure the transmission change of the sample. Special care has to be taken to ascertain optimum performance of the difference measurement when working with fluctuating light sources: (i) With the exciting pulse being blocked the intensities of the probing pulses should be adjusted by means of optical attenuators to give a signal of approximately zero at the output of the difference detection system. The energy of both probing pulses falling on the two photodiodes should be quite large. It must be adjusted to yield considerable signals without saturating the photodiodes. (ii) The absolute scale of the transmission change is readily calibrated by introducing optical attenuators of known attenuation in one of the two probing beams. (iii) The pathway of the two probing pulses should be symmetric in order to compensate fluctuations in beam direction and beam divergence. (iv) Small long-term drifts of the initial sample transmission are compensated when the same pathways of the two probing pulses in the sample are chosen. (v) The difference detector should be carefully shielded against stray light from the exciting light pulses.

\section{The difference detection system}

We now turn to the description of the difference detection system. The two photodiodes detect the incoming beam. The charges created by one pulse in the photodiode are proportional to the pulse energy. As time evolution is deduced from the optical delay scheme there is no need for a high-speed electronic circuit to measure the power of the pulses as a function of time. Consequently we may use photodetectors with large areas, which show relatively slow signal response (rise times $\simeq 10 \mathrm{~ns}$ ). The difference of the charges created by the two light pulses is formed in the difference detector (see figure 2). A subsequent impedance transformer is used to adjust the output resistance to the impedance $R=50 \Omega$ of the coaxial cable. The signal is finally digitised in the gated $\mathrm{A} / \mathrm{D}$ converter (LeCroy, model $2249 \mathrm{~W}, 11$-bit resolution, gate time used $400 \mathrm{~ns}$ ). The data of the difference channel and of a reference channel are processed in a desk-top computer, which calculates transmission changes and stores the data.

\section{The electronic layout of the difference detector}

The key element of our system is the optical difference detector. The electronic layout is shown in figure 2 . We used a pair of large-area photodetectors (Centronic OSD-50-0) with identical 


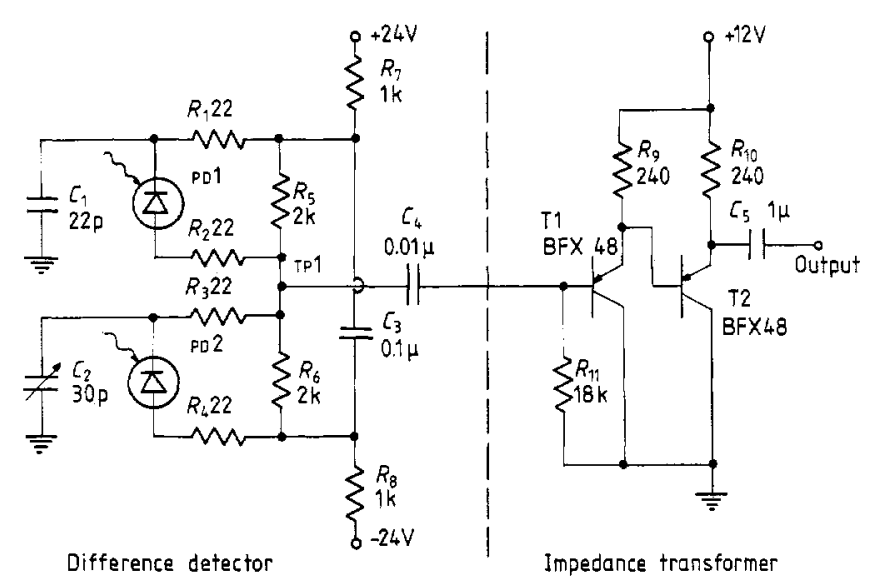

Figure 2. Circuit designed for the operation of the difference detector. Large area pin photodiodes (Centronic OSD-50-0) are used as light sensitive elements.

electrical and optical properties. The two probing pulses generate the charges $Q_{1}$ and $Q_{2}$ in the two photodetectors leading to the voltage change $\Delta U$ at the test point TP1. $\Delta U$ is proportional to the difference of the charges $Q_{1}$ and $Q_{2}$, more exactly $\int \Delta U \mathrm{~d} t \propto Q_{1}-Q_{2}$. For an equal amount of charges in both photodiodes the voltage change induced by one diode is compensated by the voltage change from the other one, i.e. only differences in the charges lead to a detectable voltage change at point TP1. The time dependence of the voltage at TP1 is determined by the resistors $R_{1}$ to $R_{6}$, capacitors $C_{1}, C_{2}$, and the intrinsic capacitance of the diodes. By adjusting capacitor $C_{2}$ we may compensate small variations of the intrinsic capacitance of the photodiodes. The resistors $R_{7}$ and $R_{8}$ are used to limit the current through the photodiodes in the case of steady-state illumination. The coupling capacitor $C_{4}$ transfers the difference signal to the impedance transformer. At its output one observes a signal with a rise time of $10 \mathrm{~ns}$ and a fall time of $200 \mathrm{~ns}$.

\section{Results}

The salient features of the difference detection system are the following: Using single picosecond pulses from a mode-locked Nd-glass laser (wavelength $\lambda=1.054 \mu \mathrm{m}$ ) with fluctuations of the pulse energy of $30 \%$ we are able to detect relative changes in the transmitted probing energy of $10^{-4}$, when averaging over ten single laser shots. The system is highly sensitive; only $10^{9}$ photons or $0.1 \mathrm{~nJ}$ per shot are required for each measurement. The experiments have shown that the accuracy of $10^{-4}$ is not limited by the nonlinearities in the electronic circuit but by the precision of the optical arrangement, especially by the fluctuations of pulse parameters such as beam direction and beam divergence. Furthermore, the system can be optimised by using more sensitive (and more expensive) photodiodes and a higher supply voltage at the photodiodes. Other types of photodetectors can be employed, provided that the difference of the signals is formed immediately behind the photosensitive elements and prior to further processing of the signal.

In summary, our difference detection system is well suited to perform time resolved transmission measurements on a picosecond time scale with very high accuracy. It has recently been used successfully in a number of experiments, where the accurate measurements of very weak changes of transmission allow the study of new ultrafast processes (Polland et al 1984, Seilmeier et al 1984).

\section{Acknowledgment}

The authors acknowledge valuable and stimulating discussions with Professor W Kaiser.

\section{References}

Polland H J, Zinth W and Kaiser W 1984 New investigations of the primary processes of bacteriorhodosin and halorhodopsin Ultrafast Phenomena IV, Springer Series in Chemical Physics 38 ed. D H Auston and K B Eisenthal (Heidelberg: SpringerVerlag) p 456

Seilmeier A, Scherer P O J and Kaiser W 1984 Ultrafast energy dissipation in solutions measured by a molecular thermometer Chem. Phys. Lett. 105140 